\title{
Determination of 8 Endogenous Alkaloid Components in Boletus Using Ultrahigh-Performance Liquid Chromatography Combined with Quadrupole-Time of Flight Mass Spectrometry
}

\author{
Junmei Ma, ${ }^{1,2}$ Qiang Li, ${ }^{2}$ Sufang Fan, ${ }^{2}$ Liangna He, ${ }^{2}$ Lei Sun, ${ }^{2}$ Di Wen, ${ }^{1}$ Hongwen Zhai, ${ }^{2}$ \\ and Yan Zhang $\mathbb{D D}^{1,2}$ \\ ${ }^{1}$ Hebei Key Laboratory of Forensic Medicine, College of Forensic Medicine, Hebei Medical University, \\ Shijiazhuang 050017, China \\ ${ }^{2}$ Hebei Food Safety Key Laboratory, Hebei Food Inspection and Research Institute, Shijiazhuang 050227, China
}

Correspondence should be addressed to Yan Zhang; snowwinglv@126.com

Received 11 July 2020; Revised 21 August 2020; Accepted 1 September 2020; Published 24 September 2020

Academic Editor: Fatma M. El-Demerdash

Copyright (c) 2020 Junmei Ma et al. This is an open access article distributed under the Creative Commons Attribution License, which permits unrestricted use, distribution, and reproduction in any medium, provided the original work is properly cited.

A ultrahigh performance liquid chromatography coupled with quadrupole-time of flight mass spectrometry (UPLC-Q-TOF/MS) method was developed for simultaneous determination of 8 endogenous alkaloid compounds in Boletus. Boletus samples were extracted by $50 \%(\mathrm{~V} / \mathrm{V})$ methanol-water solution, then separated by CORTECS UPLC HILIC column using a binary solvent system by gradient elution. The analytes were determined by Q-TOF/MS in TOF MS and information dependent acquisition (IDA)-MS/MS mode. The results showed that mass accuracy error of the 8 endogenous alkaloids were lower than $5.0 \times 10^{-6}$, good linear relationship was got in range of $0.2-500 \mu \mathrm{g} / \mathrm{L}$, and correlation coefficient was higher than 0.9990 . The limit of detection was in the range of $0.002-0.100 \mathrm{mg} / \mathrm{kg}$ and the limit of quantification was in the range of $0.004-0.200 \mathrm{mg} / \mathrm{kg}$. Recovery of the method was in range of $80.1 \%-101.5 \%$ with spike levels of $0.004-2.00 \mathrm{mg} / \mathrm{kg}$, relative standard deviations were lower than $10 \%$. The method was simple, specific, and reliable. It could be used for the rapid screening and quantitative analysis of 8 endogenous alkaloids in Boletus.

\section{Introduction}

The Boletus is an edible fungus belonging to the Fungi, Basidiomycotina, Hymenomycetes, Agaricates [1]. It is mainly distributed in Yunnan Province of China. Due to a wide variety of secondary metabolites from fungus and their biological activities, extensive attention has been paid to the researches of fungus both in China and abroad [2]. Boletus is popular because of its delicious taste, appreciated nutrition value [3], and a variety of medicinal effects [4]. The chemical composition of the Boletus was mainly terpenes, flavonoid [5], phenols [6], and alkaloids, which possessed comprehensive biological activities, such as antioxidant, antifatigue, antitumor, and anti-inflammatory [7, 8]. Generally, the species for which a particular alkaloid structure is characteristic are closely related and can be comprised within the next higher taxon, genus, or family.

Alkaloids have been reported as a group of basic organic substances of Boletus, containing at least one nitrogen atom in a ring structure in the molecule [9]. A number of alkaloids were discovered and reported with biological activities in Boletus. Nicotine is an important and most well-known alkaloid, and it is the most important alkaloid in tobacco. In addition, nicotine is also widely present in Boletus and other plants. Some countries restricted the nicotine content in some foods. For example, the European Commission stipulated that the maximum limit of nicotine in edible fungus fresh and dry products is $0.036 \mathrm{mg} / \mathrm{kg}$ and $1.17 \mathrm{mg} / \mathrm{kg}$, while the maximum limit in Boletus dried product is $2.3 \mathrm{mg} / \mathrm{kg}$. Besides nicotine, Boletus contains some important and 
pharmacologically secondary alkaloids. Choline is an indispensable substance for normal metabolism, which has the functions of promoting brain development and improving memory. Boletus is rich in amino acids. Arginine and ornithine produce putrescine under the decarboxylation of bacterial amino acid decarboxylase. Tryptamine is a monoamine alkaloid, based around the indole ring structure. It is regarded as the backbone for tryptamines, a group of compounds that include many pharmacologically active compounds, including serotonin (neurotransmitters), melatonin (hormone), and psilocybin (psychedelic drugs) [10]. Du et al. [11] isolated nicotinamide from the Boletinus pictus. Zhang et al. [12] found that anabasine, cotinine, and muscarine exist in Boletus. According to references, nicotine, anabasine, choline, tryptamine, putrescine, nicotinamide, muscarine, and cotinine can be detected in Boletus. However, due to a lack of research on the detection of alkaloids in Boletus, the inconsistency of the species, and the maturity of the tested samples, there are certain differences in the data.

At present, there are few studies on the detection of endogenous alkaloids in Boletus. The analytical methods of these alkaloids mainly include gas chromatography [13-15], high-performance liquid chromatography [16-21], and liquid chromatography-tandem mass spectrometry [22-28]. However, gas chromatography requires derivation and determination, and the process is relatively cumbersome; highperformance liquid chromatography has poor anti-interference ability [29]. Liquid chromatography-tandem mass spectrometry has a limited ability to identify isomers, and the ion dwell time is limited due to the scan rate. UPLC-Q-TOF/MS has the characteristics of high resolution, high sensitivity, high accuracy, and wide scanning range [30]. It has been widely used in quantitative analysis of pesticide residues, veterinary drug residues, and other research fields [31, 32]. In this paper, UPLC-Q-TOF/MS was first used for fast and accurate determination of endogenous alkaloids in the Boletus. It can obtain accurate and stable test results and provide reliable technical support for enterprises and regulatory authorities. This study lays the foundation for the development and establishment of quality standards in Boletus resources, provide a reference for comprehensive quality control and evaluation of Boletus and its products, and promote sustainable development of the industry.

\section{Materials and Methods}

2.1. Materials and Reagents. Methanol, acetonitrile, ethanol, and formic acid were HPLC grade and were purchased from Merck (Darmstadt, Germany). Water was purified using a Milli-Q-System (Millipore, Guyancourt, France). Ammonium acetate $\left(\mathrm{NH}_{4} \mathrm{OAc}\right)$ was purchased from Beijing Chemical Reagent Factory (Beijing, China). Formic acid was purchased from Duksan Pure Chemicals (Ansan, Korea). Eight alkaloids reference materials with purity $\geq 95 \%$ were purchased from Dr. Ehrenstorfer (Augsburg, Germany).

2.2. Instruments and Equipment. The high-speed refrigerated centrifuge (CR22N, HITACHI, Germany), the vortex mixer (Vortex Genius 3, IKA, Germany), and the ultrasonic cleaner (Elmasonic P300H, Elma, Germany) were used in the procedure of extraction. The separation of compounds was carried out on a LC-30AD UPLC system equipped with a binary solvent manager, sample manager, and column manager (Shimadzu, Japan). Quantitative analysis of target compounds was conducted on a TripleTOFTM $5600^{+}$ quadrupole/time of flight mass spectrometry ( $\mathrm{AB}$ Sciex, USA).

2.3. Sample Preparation. The sample was pulverized uniformly on a small pulverizer, and one gram of pulverized sample was weighed and transferred to a $50 \mathrm{~mL}$ centrifuge tube. Following spiking $20 \mathrm{~mL}$ of $50 \%$ methanol-water solution, vortexing for $1 \mathrm{~min}$, and sonicating for $20 \mathrm{~min}$, the sample was centrifuged at $8000 \mathrm{r} / \mathrm{min}$ for $5 \mathrm{~min}$. After centrifugation, the supernatant was filtered through a $0.22 \mu \mathrm{m}$ nylon membrane before UPLC-Q-TOF/MS detection.

2.4. Chromatographic Conditions. The chromatographic separation was performed on a CORTECS UPLC HILIC column $(100 \mathrm{~mm} \times 2.1 \mathrm{~mm}, 1.6 \mu \mathrm{m}$; Waters, USA $)$. The column temperature was $40^{\circ} \mathrm{C}$. The injection volume was $5.0 \mu \mathrm{L}$. The flow rate was $300 \mu \mathrm{L} / \mathrm{min}$. $20 \mathrm{mmol} / \mathrm{L} \mathrm{NH}_{4} \mathrm{OAc}$ solution containing $0.1 \%(\mathrm{v} / \mathrm{v})$ formic acid (phase A) and acetonitrile containing $0.1 \%(\mathrm{v} / \mathrm{v})$ formic acid (phase B) were used as mobile phase. The consecutive program was as follows: $0-3.00 \mathrm{~min}, 85 \% \mathrm{~B}$; $3.0-6.0 \mathrm{~min}, 85 \%$ to $60 \% \mathrm{~B}$; $6.0-11.0 \mathrm{~min}, 60 \% \mathrm{~B} ; 11.0-12.0 \mathrm{~min}, 60 \%$ to $85 \% \mathrm{~B}$; $12.0-15.0 \mathrm{~min}, 85 \% \mathrm{~B}$.

2.5. Mass Spectrometry Conditions. The MS analysis was performed using an electrospray ion source (ESI) in positive ionization mode. The optimized parameters of ion source were as follows: the ionization voltage was $5.5 \mathrm{kV}$, the source temperature was $400^{\circ} \mathrm{C}$, the pressure of curtain gas was 35 psi, the pressure of nebulizer gas was $50 \mathrm{psi}$, and the pressure of auxiliary gas was $55 \mathrm{psi}$; TOF MS conditions were as follows: scan range was $50 \sim 500 \mathrm{~m} / \mathrm{z}$, duration time was $15 \mathrm{~min}$, and accumulation time was $0.15 \mathrm{~s}$. IDA-MS/MS conditions were as follows: accumulation time was $0.05 \mathrm{~s}$, high sensitivity mode was set, exclude isotopes were within $4 \mathrm{Da}$. Declustering potential was $80 \mathrm{~V}$, collision energy was $35 \mathrm{~V}$, and the dynamic energy of collision was $15 \mathrm{eV}$. Before each experiment, the instrument performed mass accuracy calibration through the CDS system. During the experiment, every five samples were run, and the mass accuracy calibration was automatically performed. The mass spectrum information of 8 endogenous alkaloids was shown in Table 1.

2.6. Optimization of Sample Extraction. To find out an optimum extraction method for samples, this experiment investigated the extraction effect when acetonitrile, methanol, ethanol, water, $50 \%$ acetonitrile-water solution, $50 \%$ methanol-water solution, and $50 \%$ ethanol-water solution were used as the extraction solvent, and the extraction time of $10,15,20$, and 30 min was compared. 
TABLE 1: Mass parameters for the 8 endogenous alkaloids.

\begin{tabular}{|c|c|c|c|c|c|c|}
\hline Compound name & Formula & Adduct/charge & $\begin{array}{l}\text { Theoretical mass } \\
(\mathrm{m} / \mathrm{z})\end{array}$ & $\begin{array}{c}\text { Experimental mass } \\
(\mathrm{m} / \mathrm{z})\end{array}$ & Mass error $\left(\times 10^{-6}\right)$ & Retention time (min) \\
\hline Nicotine & $\mathrm{C}_{10} \mathrm{H}_{14} \mathrm{~N}_{2}$ & {$[\mathrm{M}+\mathrm{H}]^{+}$} & 163.123 & 163.1231 & 0.9 & 4.69 \\
\hline Anabasine & $\mathrm{C}_{10} \mathrm{H}_{12} \mathrm{~N}_{2}$ & {$[\mathrm{M}+\mathrm{H}]^{+}$} & 161.107 & 161.1069 & -2.5 & 2.97 \\
\hline Choline & $\mathrm{C}_{5} \mathrm{H}_{14} \mathrm{NO}^{+}$ & {$[\mathrm{M}+\mathrm{H}]^{+}$} & 104.107 & 104.1071 & 1.5 & 4.30 \\
\hline Tryptamine & $\mathrm{C}_{10} \mathrm{H}_{12} \mathrm{~N}_{2}$ & {$[\mathrm{M}+\mathrm{H}]^{+}$} & 161.107 & 161.1075 & 0.9 & 1.80 \\
\hline Putrescine & $\mathrm{C}_{4} \mathrm{H}_{12} \mathrm{~N}_{2}$ & {$[\mathrm{M}+\mathrm{H}]^{+}$} & 89.107 & 89.1073 & -0.1 & 6.55 \\
\hline Nicotinamide & $\mathrm{C}_{6} \mathrm{H}_{6} \mathrm{~N}_{2} \mathrm{O}$ & {$[\mathrm{M}+\mathrm{H}]^{+}$} & 123.055 & 123.0554 & 0.8 & 1.04 \\
\hline Muscarine & $\mathrm{C}_{9} \mathrm{H}_{20} \mathrm{NO}_{2}^{+}$ & {$[\mathrm{M}+\mathrm{H}]^{+}$} & 174.149 & 174.1491 & 0.6 & 3.79 \\
\hline Cotinine & $\mathrm{C}_{10} \mathrm{H}_{12} \mathrm{~N}_{2} \mathrm{O}$ & {$[\mathrm{M}+\mathrm{H}]^{+}$} & 177.102 & 177.1025 & 1.4 & 1.34 \\
\hline
\end{tabular}

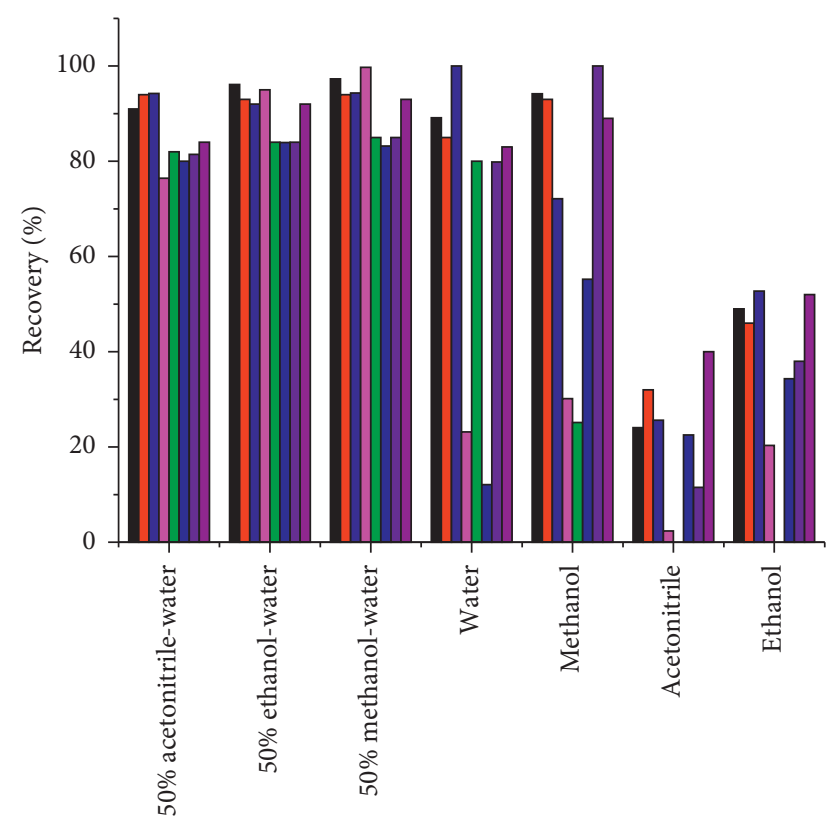

Extraction solvent
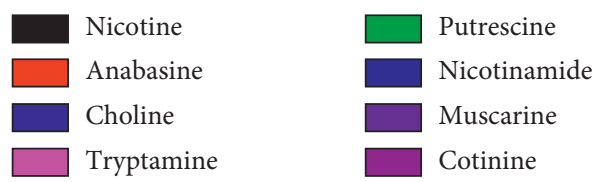

FIGURE 1: Effect of different solvent on the recoveries of 8 endogenous alkaloids.

2.7. Optimization of Liquid Chromatography and Mass Spectrometry Conditions. Three analytical columns were tested prior to the selection of any additional chromatographic parameters, the Acquity BEH C18 column $(100 \mathrm{~mm} \times 2.1 \mathrm{~mm}, 2.5 \mu \mathrm{m})$, the Acquity UPLC HSS T3 column $(100 \mathrm{~mm} \times 2.1 \mathrm{~mm}, 1.8 \mu \mathrm{m})$, and the Cortecs UPLC HILIC column $(100 \mathrm{~mm} \times 2.1 \mathrm{~mm}, 1.6 \mu \mathrm{m})$ were used. The mobile phase was evaluated. First, methanol and acetonitrile were tested as organic solvents in the mobile phase. In relation to the aqueous phase, an aqueous solution with various concentrations of $\mathrm{NH}_{4} \mathrm{OAc}(0,5 \mathrm{mmol} / \mathrm{L}, 10 \mathrm{mmol} / \mathrm{L}$, $15 \mathrm{mmol} / \mathrm{L}, 20 \mathrm{mmol} / \mathrm{L}$ ) were tested.

Syringe injection was used to inject 8 endogenous alkaloids directly into the mass spectrometer. The experiment investigated the response of target compounds under positive and negative ionization modes. For this method, TOF

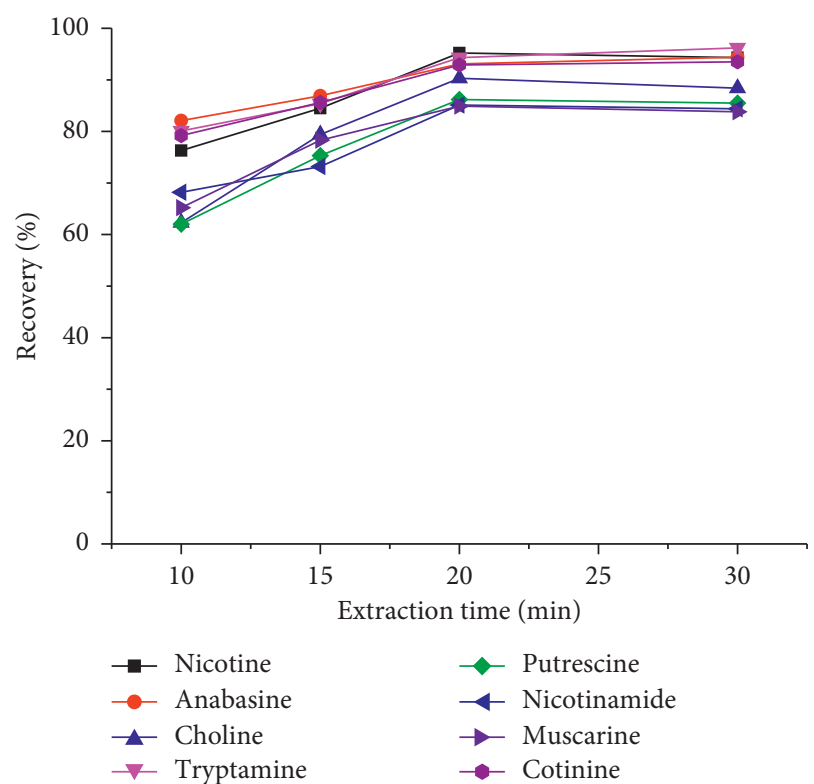

Figure 2: Comparison of extraction efficiency at extraction times.

MS and IDA MS/MS modes were adopted for the detection of a target analyte. Under TOF MS mode, the experiment investigated the response of target compounds under different declustering potentials $(50-300 \mathrm{~V})$ and found the declustering potentials with the highest response.

2.8. Method Validation. Validation of the whole analytical method was performed with linearity, the limit of detection (LOD), the limit of quantification (LOQ), precision, accuracy, and recovery. The linearity of the method was evaluated by constructing calibration curves with different concentrations of 8 alkaloids. The LOD and LOQ under the present chromatographic conditions were calculated on the basis of the response and slope of each regression equation at signalto-noise ratios $(\mathrm{S} / \mathrm{N})$ of $3: 1$ and $10: 1$, respectively. The recoveries of analytes were evaluated by adding the standard solutions with three different concentration levels $(1 x$ LOQ, $3 x$ LOQ, 10x LOQ) to the known amounts of fungus samples, and each level was repeated six times. The precision of the method was expressed by a relative standard deviation (RSD). 


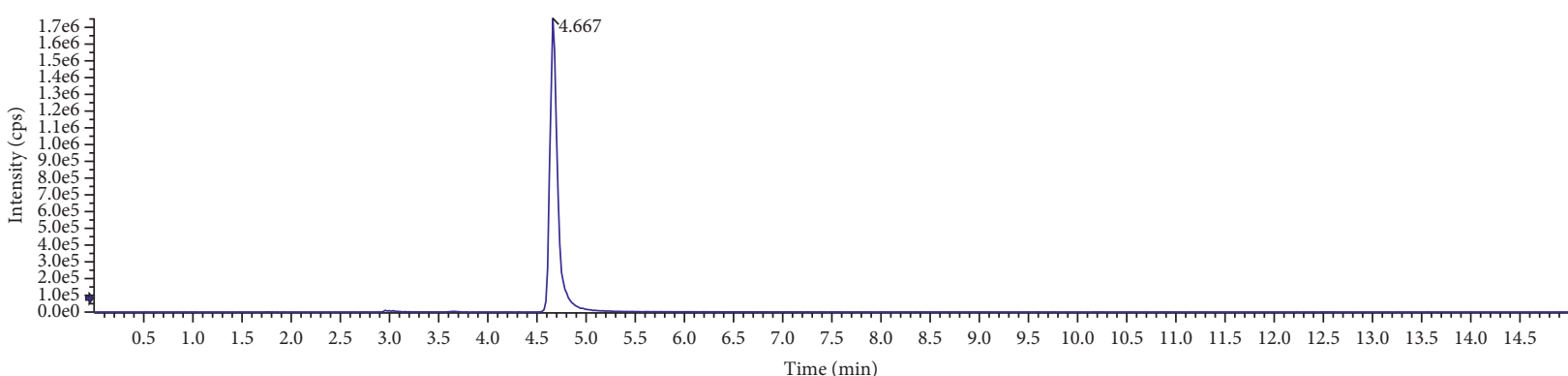

(a)

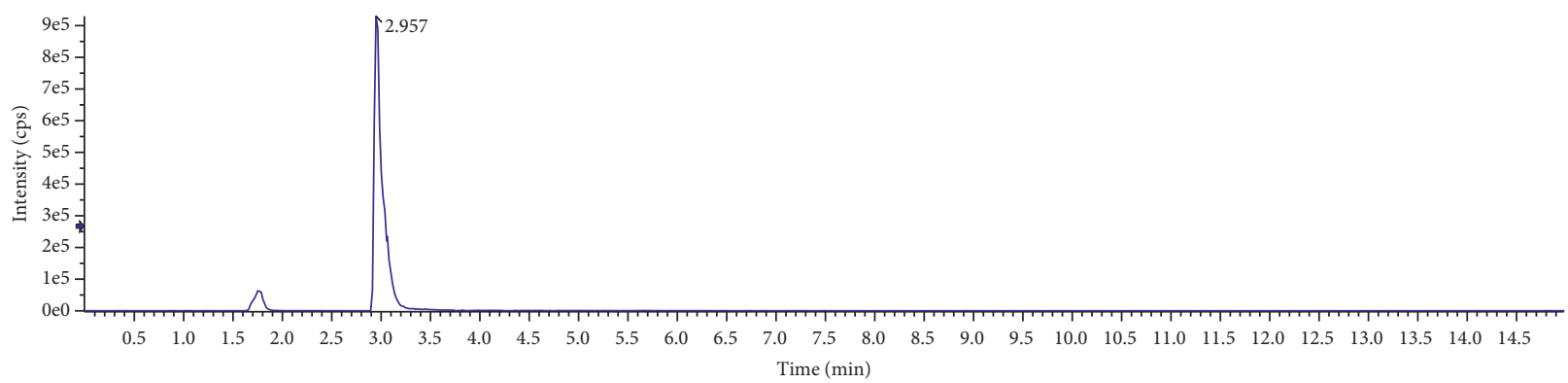

(b)

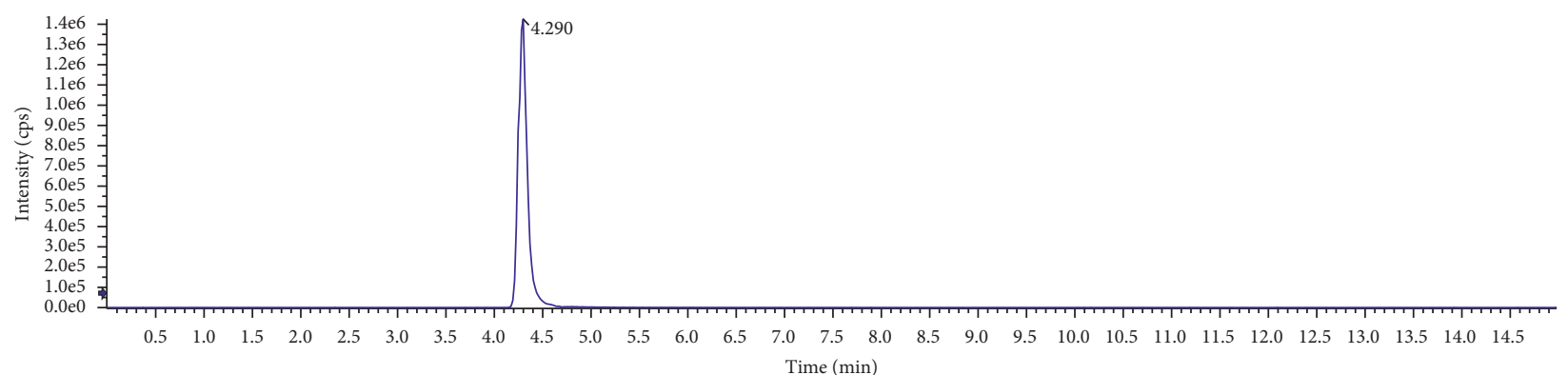

(c)

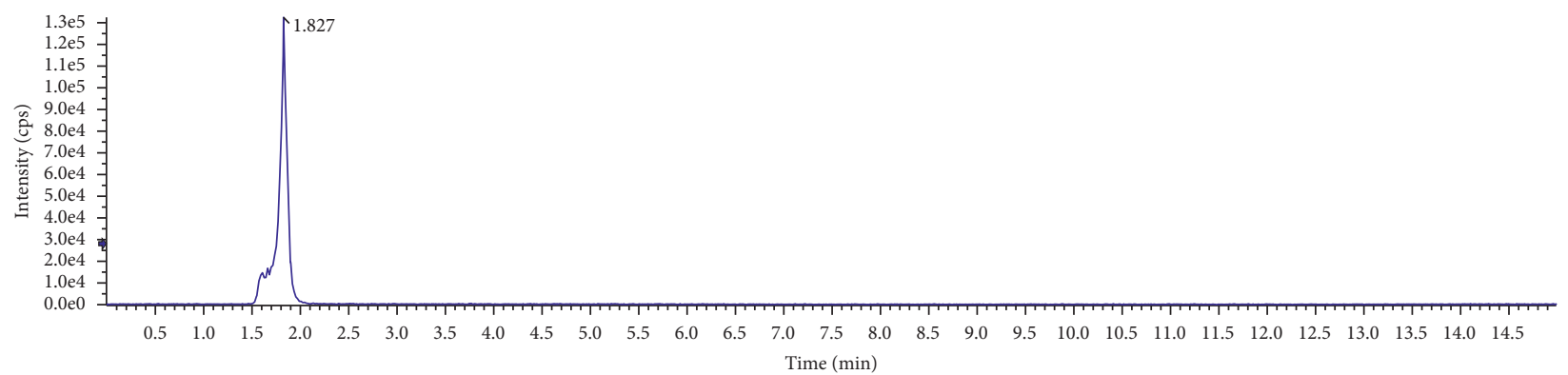

(d)

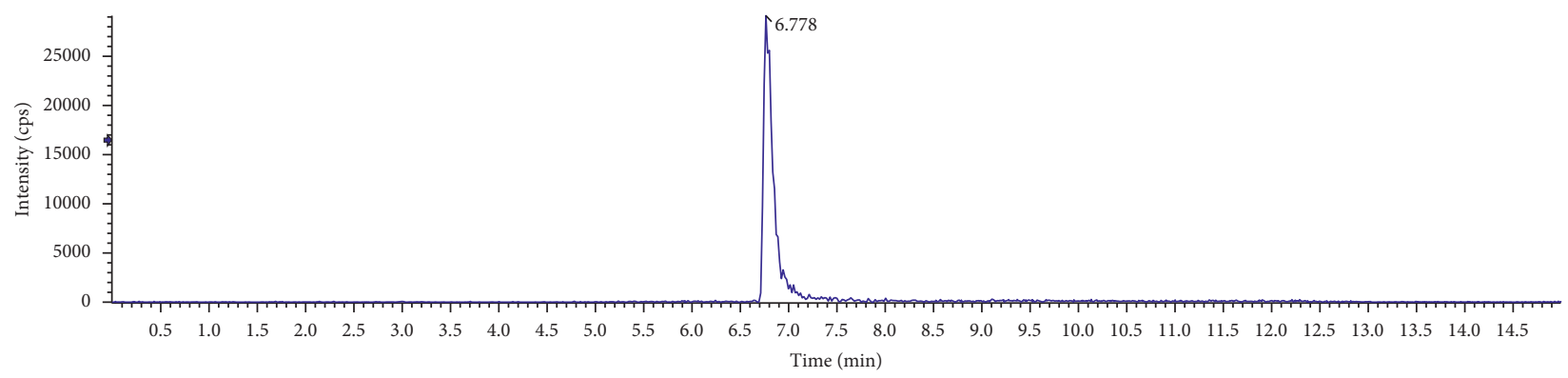

(e)

Figure 3: Continued. 


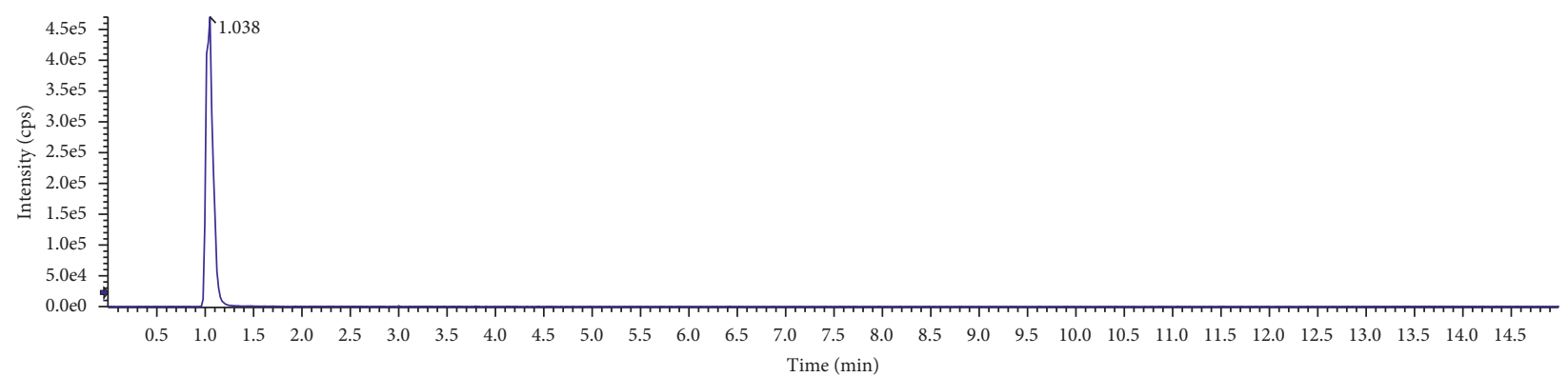

(f)

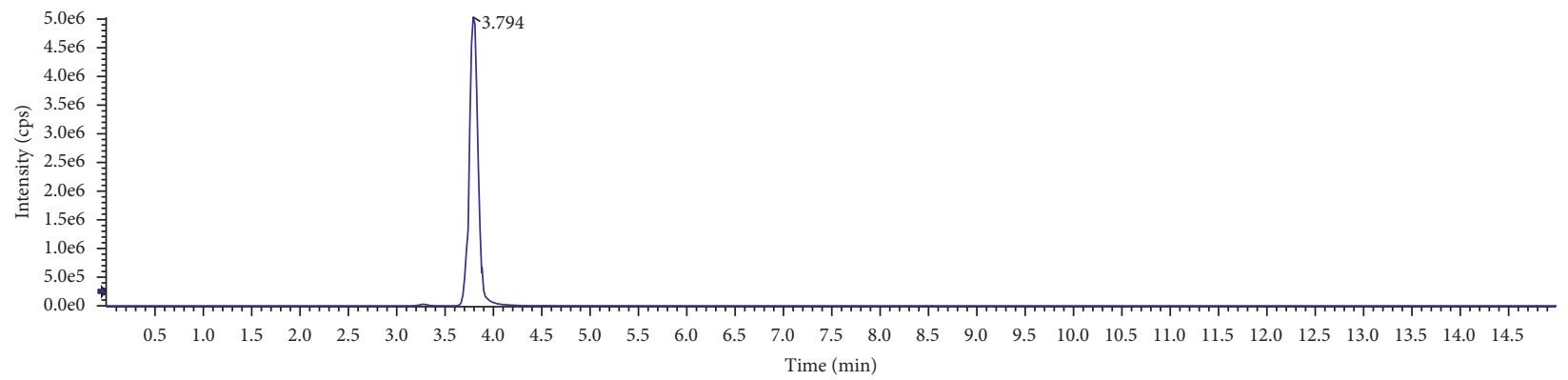

(g)

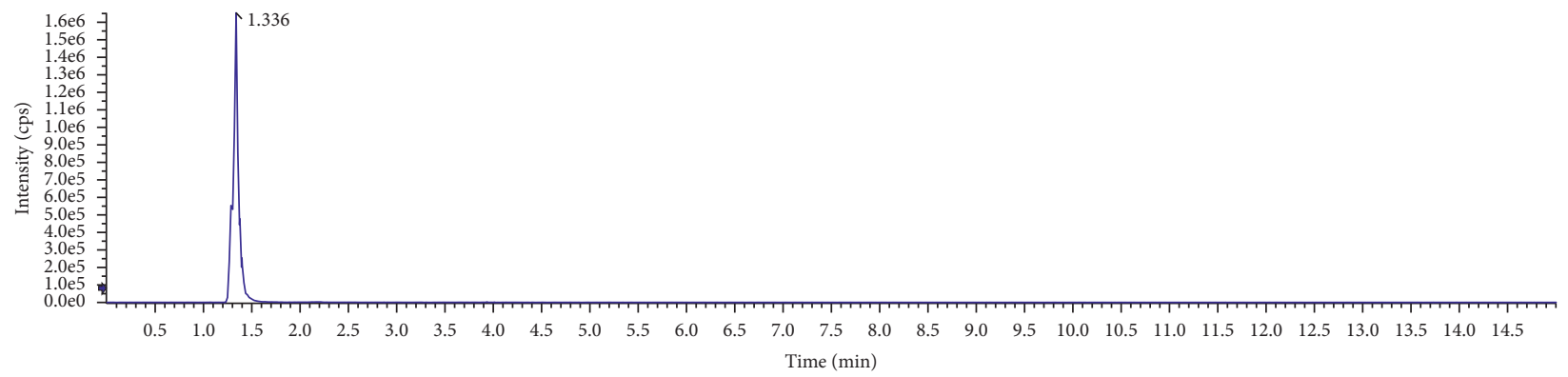

(h)

Figure 3: Extracted ion chromatograms of the 8 endogenous alkaloids. (a) Nicotine. (b) Anabasine. (c) Choline. (d) Tryptamine. (e) Putrescine. (f) Nicotinamide. (g) Muscarine. (h) Cotinine.

\section{Results and Discussion}

\subsection{Optimization of Sample Extraction}

3.1.1. Optimization of Extraction Solvents. As Figure 1 shows, the extraction efficiency of extraction solvents for various endogenous alkaloids was different. 50\% methanolwater solution yielded the best reproducibility and recovery of the 8 alkaloids. Therefore, a 50\% methanol-water solution was selected as the extraction solvent.

3.1.2. Optimization of Extraction Times. As Figure 2 shows, when the ultrasonic extraction time was increased from $10 \mathrm{~min}$ to $20 \mathrm{~min}$, the extraction efficiency of alkaloids was greatly improved. After $20 \mathrm{~min}$, the growth trend decelerates, and the extraction efficiency of alkaloids changes little. Considering the extraction efficiency of the experiment and time cost, $20 \mathrm{~min}$ was chosen as extraction time.

\subsection{Optimization of Liquid Chromatography and Mass Spectrometry Conditions}

3.2.1. Optimization of Liquid Chromatography Conditions. The results showed that the nicotine, anabasine, choline, tryptamine, putrescine, and muscarine were not retained on the Acquity $\mathrm{BEH} \mathrm{C}_{18}$ column and the Acquity UPLC HSS T3 column. They can be retained on the CORTECS UPLC HILIC column, and the separation effect was good. Because they are strong polar hydrophilic compounds, which were weakly retained on the $\mathrm{C}_{18}$ and $\mathrm{T} 3$ columns, so it is necessary to replace hydrophilic columns for separation. Therefore, the CORTECS UPLC HILIC column $(100 \mathrm{~mm} \times 2.1 \mathrm{~mm}$, $1.6 \mu \mathrm{m})$ was selected as the analytical columns.

The mobile phase was evaluated. First, methanol and acetonitrile were tested as organic solvents in the mobile phase, observing that methanol and acetonitrile as mobile phases have little effect on the signal intensity, but when methanol was used as the mobile phase for gradient elution, column pressure varies widely, and equilibration takes 


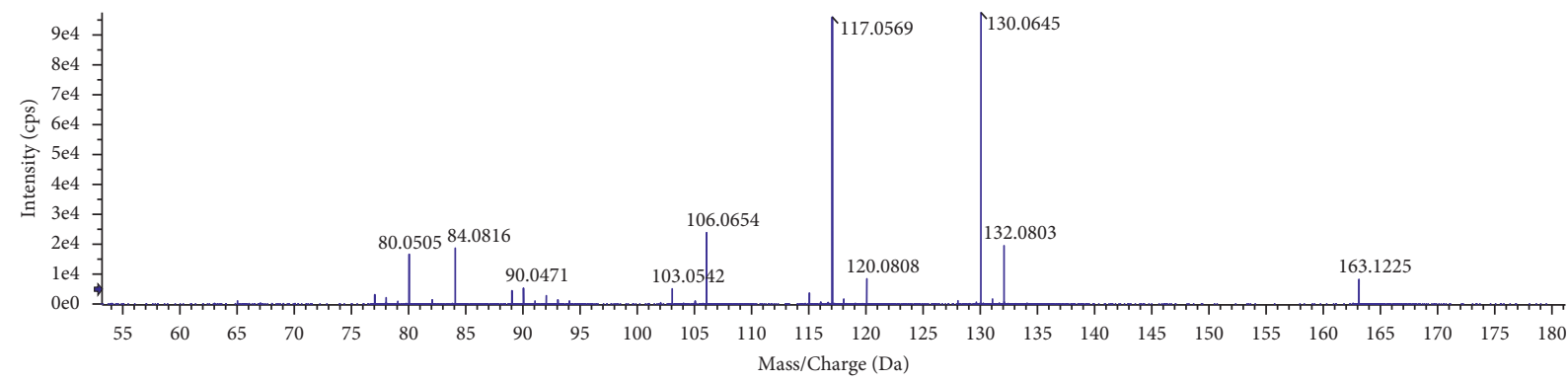

(a)

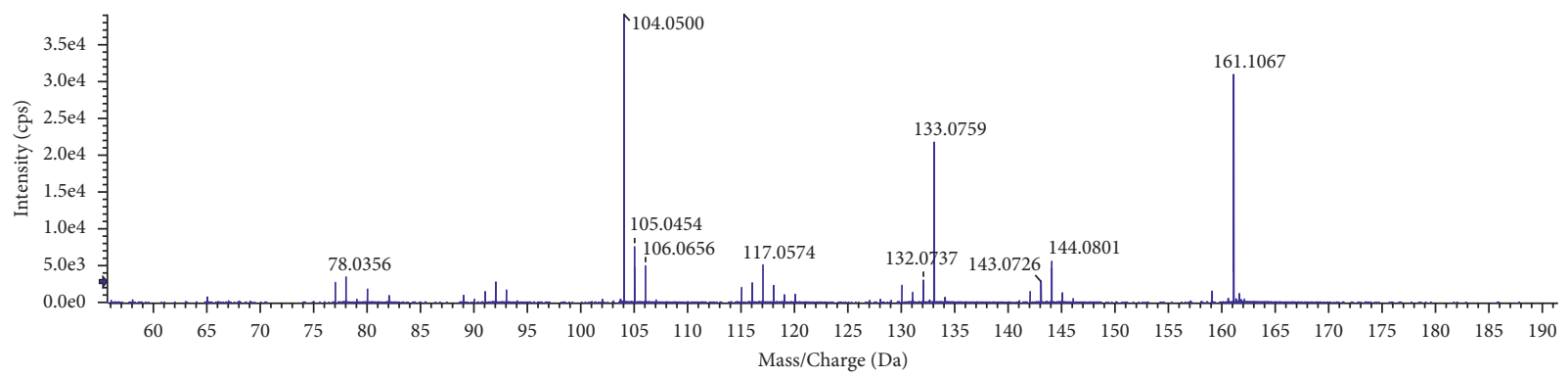

(b)

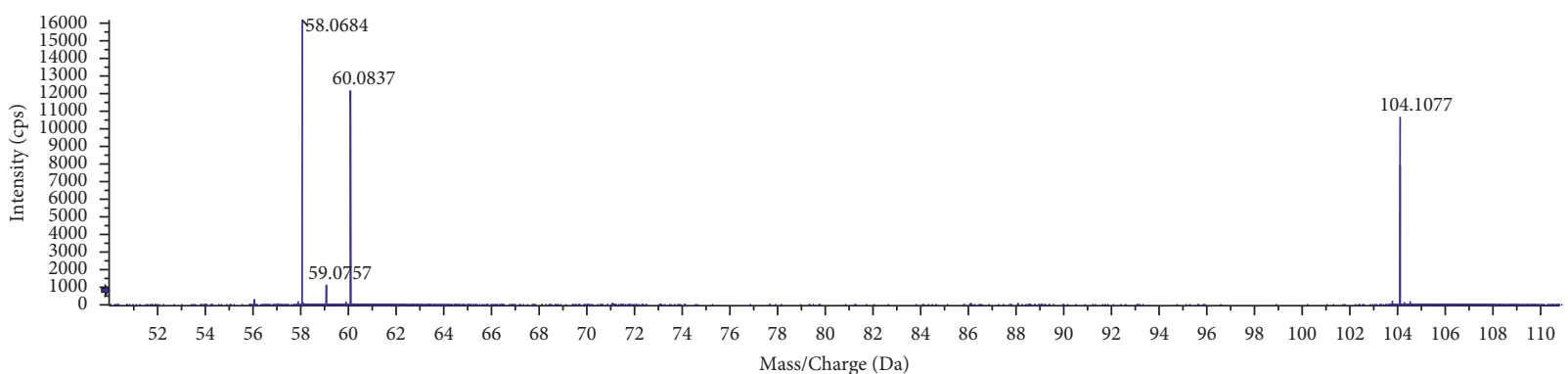

(c)

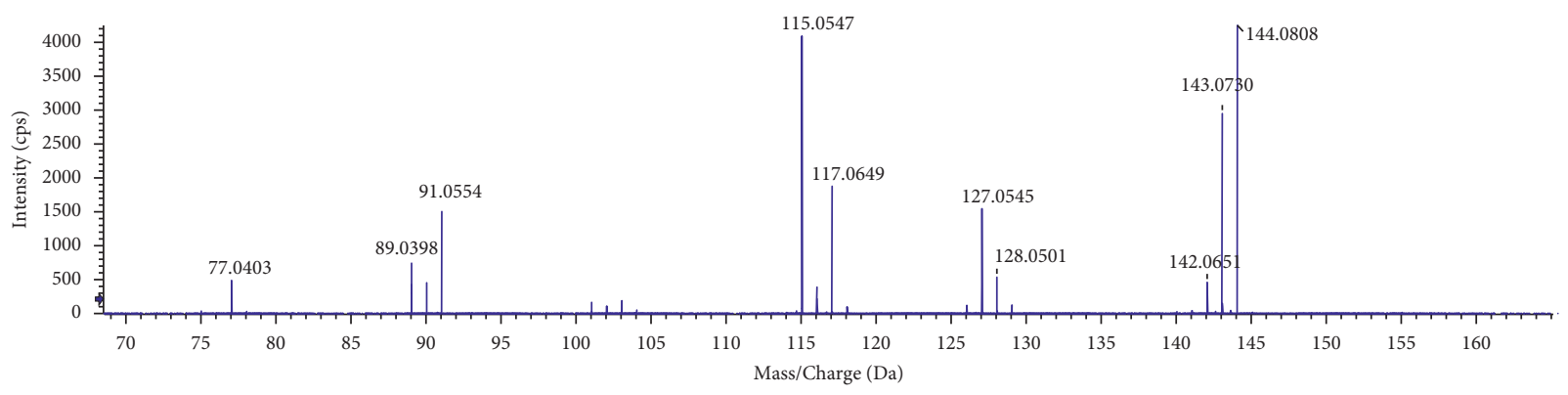

(d)

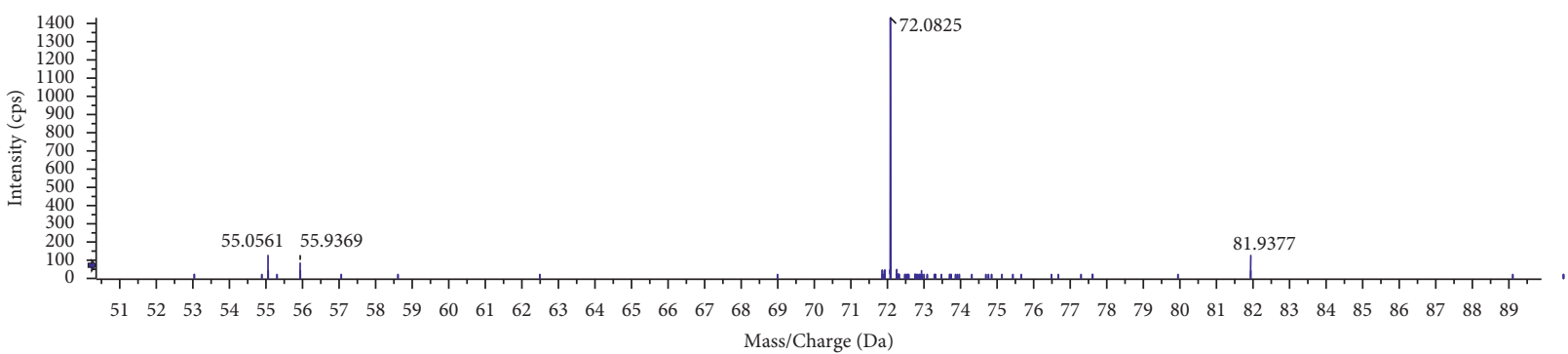

(e)

FIgure 4: Continued. 


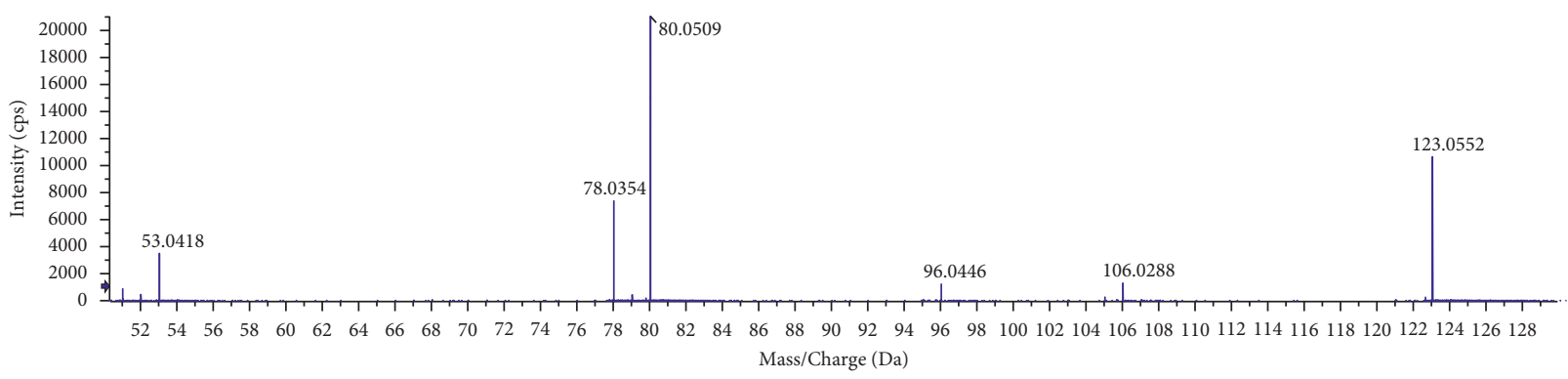

(f)

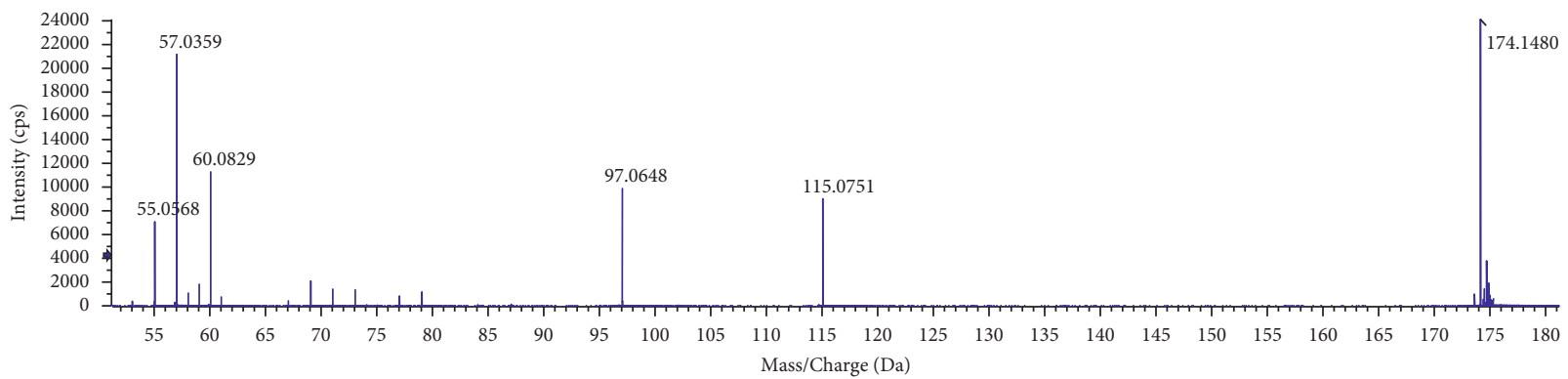

(g)

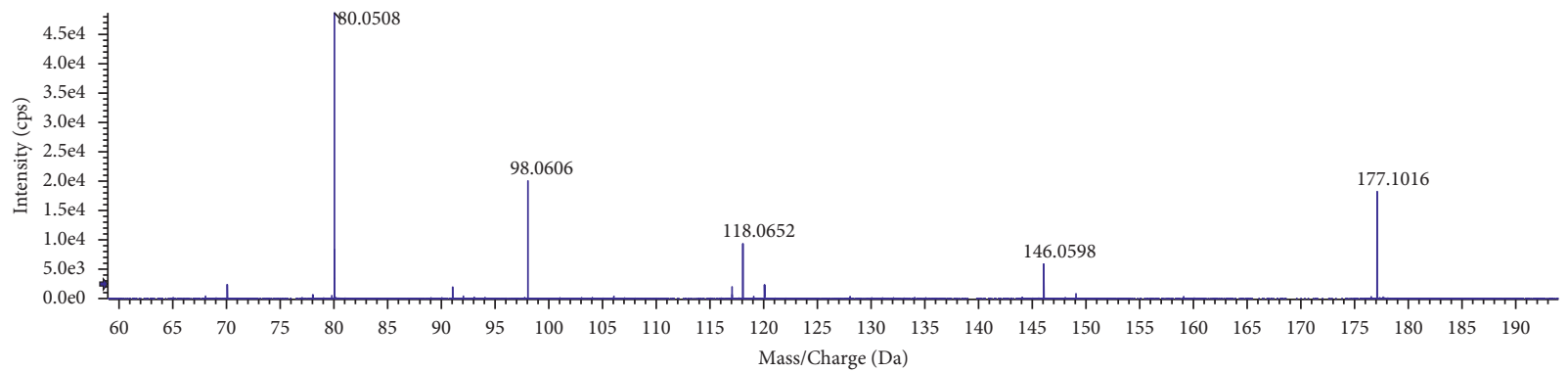

(h)

Figure 4: MS/MS spectra of the 8 endogenous alkaloids. (a) Nicotine. (b) Anabasine. (c) Choline. (d) Tryptamine. (e) Putrescine. (f) Nicotinamide. (g) Muscarine. (h) Cotinine.

longer. In relation to the aqueous phase, the results showed $20 \mathrm{mmol} / \mathrm{L} \mathrm{NH}_{4} \mathrm{OAc}$ as the aqueous solution showed better separation and elution capabilities. Considering that under the positive mode, the addition of formic acid could increase the ionization of the compounds, which improved the separation efficiency and the intensity of the mass spectrometry signal. Therefore, $20 \mathrm{mmol} / \mathrm{L} \mathrm{NH} \mathrm{NH}_{4} \mathrm{Oc}$ solution containing $0.1 \%(\mathrm{v} / \mathrm{v})$ formic acid and acetonitrile containing $0.1 \%(\mathrm{v} / \mathrm{v})$ formic acid were selected as the mobile phase for subsequent experiments.

3.2.2. Optimization of Mass Spectrometry Parameters. The TripleTOF ${ }^{\mathrm{TM}} 5600^{+}$instrument has a CDS automatic calibration infusion system. The reference spray of the DuoSpray $^{\text {тм }}$ ion source was used to input the calibration solution for automatic system calibration. The DuoSpray ${ }^{\mathrm{TM}}$ ion source has two types: ESI and atmospheric pressure chemical ionization source (APCI). In this experiment, the ESI was selected as the detection ion source, and the APCI was used as the calibration ion source. Through the automatic calibration system, automatic batch calibration was performed to ensure the accurate mass of the system was stable for a long time.
The results showed that compounds have a higher response in positive ionization mode, so the positive ionization mode was used for detection. Under TOF MS mode, the experiment investigated the response of target compounds under different declustering potentials (50-300 V), with the findings that at the fragmentor voltage of $80 \mathrm{~V}$, compound response was the highest; relatively low fragmentor voltage was unfavorable for ion transmission, and overly high fragmentor voltage would cause the compound to fragment within the source. The accurate mass, retention time, and isotope ratio was obtained. Figure 3 shows the extracted ion chromatograms of 8 alkaloids $(500 \mathrm{ng} / \mathrm{mL})$. The accurate mass deviations of target compounds were less than $5.0 \times 10^{-6}$ (Table 1 ). The MS/MS spectra of the target were used for the final confirmation of the initial screening results of the accurate mass (Figure 4).

\subsection{Method Validation}

3.3.1. Linearity and Sensitivity. As Table 2 shows, the linear range was studied by preparing a calibration curve with a concentration range of $0.2-500 \mu \mathrm{g} / \mathrm{L}$ for each compound, 
TABLE 2: Linearity, LODs, and LOQs of the 8 endogenous alkaloids.

\begin{tabular}{|c|c|c|c|c|c|}
\hline Compound & Linear equation & Linear range $(\mu \mathrm{g} / \mathrm{L})$ & $R^{2}$ & $\mathrm{LOD}(\mathrm{mg} / \mathrm{kg})$ & LOQ (mg/kg) \\
\hline Nicotine & $Y=10091.33111 X+3329.87164$ & $0.5-500$ & 0.99866 & 0.005 & 0.010 \\
\hline Anabasine & $Y=11037.04373 X+23487.04699$ & $0.2-500$ & 0.99844 & 0.002 & 0.004 \\
\hline Choline & $Y=14658.46420 X-5.77788 \times 10^{4}$ & $0.5-500$ & 0.99785 & 0.005 & 0.010 \\
\hline Tryptamine & $Y=946.35326 X+3594.74788$ & $5.0-500$ & 0.99876 & 0.050 & 0.100 \\
\hline Putrescine & $Y=483.76213 X+2103.10951$ & $10.0-500$ & 0.99876 & 0.100 & 0.200 \\
\hline Nicotinamide & $Y=2672.35197 X-3753.02170$ & $0.5-500$ & 0.99899 & 0.005 & 0.010 \\
\hline Muscarine & $Y=28863.69968 X-3.26291 \times 10^{5}$ & $0.2-500$ & 0.99932 & 0.002 & 0.004 \\
\hline Cotinine & $Y=6622.74425 X+3.13607 \times 10^{4}$ & $0.5-500$ & 0.99928 & 0.005 & 0.010 \\
\hline
\end{tabular}

TABLe 3: Linearity, LODs, and LOQs of the 8 endogenous alkaloids.

\begin{tabular}{|c|c|c|c|c|}
\hline Compound & Background (mg/kg) & Added $(\mathrm{mg} / \mathrm{kg})$ & Recovery (\%) & RSD (\%) \\
\hline & & 0.010 & 87.6 & 1.93 \\
\hline \multirow[t]{3}{*}{ Nicotine } & 0.160 & 0.030 & 85.2 & 3.90 \\
\hline & & 0.100 & 90.4 & 1.62 \\
\hline & & 0.004 & 101.5 & 4.15 \\
\hline \multirow[t]{3}{*}{ Anabasine } & 0.111 & 0.012 & 85.9 & 0.30 \\
\hline & & 0.040 & 81.1 & 1.63 \\
\hline & & 0.010 & 89.2 & 1.45 \\
\hline \multirow[t]{3}{*}{ Choline } & 301 & 0.030 & 92.3 & 0.44 \\
\hline & & 0.100 & 88.7 & 0.13 \\
\hline & & 0.100 & 83.3 & 2.10 \\
\hline \multirow[t]{3}{*}{ Tryptamine } & ND & 0.300 & 82.4 & 6.82 \\
\hline & & 1.00 & 82.3 & 1.89 \\
\hline & & 0.200 & 80.1 & 7.59 \\
\hline \multirow[t]{3}{*}{ Putrescine } & ND & 0.600 & 89.2 & 3.08 \\
\hline & & 2.00 & 88.3 & 4.21 \\
\hline & & 0.010 & 82.5 & 3.38 \\
\hline \multirow[t]{3}{*}{ Nicotinamide } & 7.01 & 0.030 & 80.1 & 2.58 \\
\hline & & 0.100 & 91.7 & 1.43 \\
\hline & & 0.004 & 97.5 & 3.45 \\
\hline \multirow[t]{3}{*}{ Muscarine } & ND & 0.012 & 91.3 & 1.68 \\
\hline & & 0.040 & 88.8 & 0.06 \\
\hline & & 0.010 & 85.4 & 4.54 \\
\hline \multirow[t]{2}{*}{ Cotinine } & ND & 0.030 & 87.3 & 7.58 \\
\hline & & 0.100 & 90.5 & 2.19 \\
\hline
\end{tabular}

*ND means not detected.

TABLE 4: Content of the 8 endogenous alkaloids in actual samples.

\begin{tabular}{lccc}
\hline Compound & Boletus albus peck & Boletus rubellus krombh & Boletus impolitus \\
\hline Nicotine $(\mathrm{mg} / \mathrm{kg})$ & 0.491 & 1.45 & 3.41 \\
Anabasine $(\mathrm{mg} / \mathrm{kg})$ & $\mathrm{ND}$ & $\mathrm{ND}$ & $\mathrm{ND}$ \\
Choline $(\mathrm{mg} / \mathrm{kg})$ & 147 & 131 & 142 \\
Tryptamine $(\mathrm{mg} / \mathrm{kg})$ & 115 & 9.15 & 45.0 \\
Putrescine $(\mathrm{mg} / \mathrm{kg})$ & 108 & 90.6 & 8.6 \\
Nicotinamide $(\mathrm{mg} / \mathrm{kg})$ & 9.34 & 6.68 & 3.94 \\
Muscarine $(\mathrm{mg} / \mathrm{kg})$ & 0.215 & 0.0329 & 0.0483 \\
Cotinine $(\mathrm{mg} / \mathrm{kg})$ & $\mathrm{ND}$ & $\mathrm{ND}$ & $\mathrm{ND}$ \\
\hline
\end{tabular}

and a good linear relationship with correlation coefficients $\left(R^{2}\right)$ higher than 0.9990 was achieved for 8 endogenous alkaloids in their respective linear range. The LODs of 8 endogenous alkaloids were in the range of $0.002-0.100 \mathrm{mg} / \mathrm{kg}$.
The LOQs of 8 endogenous alkaloids were in the range of $0.004-0.200 \mathrm{mg} / \mathrm{kg}$. LOD and LOQ for the methods of determination of 8 endogenous alkaloids in the tea are shown in Table 2. 
3.3.2. Recovery and Precision. The recoveries of analytes were evaluated by adding the standard solutions with three different concentration levels to the known amounts of fungus samples. The data of recovery and precision are given in Table 3; the average recoveries of 8 endogenous alkaloids were in the range between $80.1 \%$ and $101.5 \%$. The RSDs were in the range of $0.06 \%-7.59 \%$.

3.4. Application to Actual Samples. In order to investigate the content of 8 endogenous alkaloids in Boletus, three Boletus samples from the local supermarket were analyzed using the developed method in this study. Their detection results are shown in Table 4. The compositions and contents of alkaloids were different in 3 Boletus samples. Anabasine and cotinine were not detected. The contents of muscarine in 3 Boletus samples were the lowest, which were 0.215, 0.0329, and $0.0483 \mathrm{mg} / \mathrm{kg}$, respectively. The contents of choline in 3 Boletus samples were the highest, which were 147, 131, and $142 \mathrm{mg} / \mathrm{kg}$, respectively.

\section{Conclusions}

In this experiment, a rapid and sensitive UPLC-Q-TOF/MS method was developed to analyze 8 endogenous alkaloids in the Boletus sample. The analytes were determined by Q-TOF/MS in TOF MS and IDA-MS/MS mode. In TOF MS mode, the target compounds qualified by the retention time, accurate mass, isotope distribution, and isotope abundance ratio of the target, and quantitated by the peak area of the excimer ion peak. In IDA-MS/MS mode, the target compounds were further confirmed by the ion fragment information under the corresponding collision energy. The linearity, sensitivity, accuracy, and precision of the method were investigated. The method has simple sample processing, high sensitivity, and high analysis efficiency. It is suitable for the rapid detection of alkaloids in batches of Boletus samples and can be used for quality control and formulation experiments of Boletus production. This method also provides a reference for the determination of various alkaloids in tobacco, tea, and other samples.

\section{Data Availability}

The data used to support the findings of this study are included within the article.

\section{Conflicts of Interest}

The authors declare that they have no conflicts of interest.

\section{Authors' Contributions}

YZ was responsible for the conceptualization of the study; JM, QL, SF, LH, LS, DW, and HZ investigated the study; JM, QL, LH, LS, and HZ reviewed the study; JM, SF, and DW were involved in the discussion; JM and YZ were responsible for writing, reviewing, and editing the original draft; YZ was involved in the project administration and funding acquisition.

\section{Acknowledgments}

This work was supported by the National Key Research Project (2019YFC1606400), National Key Research Project of Hebei Province (20375502D) and the Open Project of National R\&D Center for Edible Fungus Processing Technology (20200109).

\section{References}

[1] A. Choma, K. Nowak, I. Komaniecka et al., "Chemical characterization of alkali-soluble polysaccharides isolated from a Boletus edulis (Bull.) fruiting body and their potential for heavy metal biosorption," Food Chemistry, vol. 266, no. 15, pp. 329-334, 2018.

[2] L. Zhang, Y. Hu, X. Duan et al., "Characterization and antioxidant activities of polysaccharides from thirteen Boletus mushrooms," International Journal of Biological Macromolecules, vol. 113, no. 1, pp. 1-7, 2018.

[3] Y. Li, L. You, F. Dong, W. Yao, and J. Chen, "Structural characterization, antiproliferative and immunoregulatory activities of a polysaccharide from Boletus Leccinum rugosiceps," International Journal of Biological Macromolecules, vol. 157, no. 15, pp. 106-118, 2020.

[4] N.-N. Yang, S.-Z. Huang, Q.-Y. Ma et al., "A new pyrrole alkaloid from leccinum extremiorientale," Chemistry of Natural Compounds, vol. 51, no. 4, pp. 730-732, 2015.

[5] M. Gasecka, P. Rzymski, M. Mleczek et al., "The relationship between metal composition, phenolic acid and flavonoid content in Imleria badia from non-polluted and polluted areas," Journal of Environmental Science and Health Part B-Pesticides Food Contaminants and Agricultural Wastes, vol. 52, no. 3, pp. 171-177, 2017.

[6] I. K. Jedidi, I. K. Ayoub, T. Philippe, and N. Bouzouita, "Chemical composition and nutritional value of three Tunisian wild edible mushrooms," Journal of Food Measurement and Characterization, vol. 11, no. 4, pp. 2069-2075, 2017.

[7] P. Ginterová, B. Sokolová, P. Ondra et al., "Determination of mushroom toxins ibotenic acid, muscimol and muscarine by capillary electrophoresis coupled with electrospray tandem mass spectrometry," Talanta, vol. 125, pp. 242-247, 2014

[8] S. Vidovic, I. O. Mujic, Z. Zekovic, and V. T. Saponjac, "Antioxidant properties of selected boletus mushrooms," Food Biophysics, vol. 5, no. 1, pp. 49-58, 2010.

[9] P. Kittakoop, C. Mahidol, and S. Ruchirawat, "Alkaloids as important scaffolds in therapeutic drugs for the treatments of cancer, tuberculosis, and smoking cessation," Current Topics in Medicinal Chemistry, vol. 14, no. 2, pp. 239-252, 2013.

[10] Z. A. Mahmood, S. W. Ahmed, L. Azhar et al., "Bioactive alkaloids produced by fungi. I. Updates on alkaloids from the species of the genera Boletus, Fusarium and psilocybe," Pakistan Journal of Pharmaceutical Sciences, vol. 23, no. 3, pp. 349-357, 2010.

[11] Z. W. Du, J. K. Liu, C. Xiang, and G. Wang, "Chemical constituents of Boletinus pictus," Natural Product Research and Development, vol. 24, no. 5, pp. 618-621, 2012.

[12] H. F. Zhang, Y. B. Luo, X. Y. Li et al., "Determination of nicotine and secondary alkaloids in edible plants using modified QuEChERS procedure coupled with gas chromatography/mass spectrometry," Journal of Analytical Science, vol. 34, no. 2, pp. 239-244, 2018.

[13] M. Woniakiewicz, A. Woźniakiewicz, P. M. Nowak et al., "“Green" and complementary methods for the analysis of biogenic amines in wine," Food Analytical Methods, vol. 11, pp. 2614-2627, 2018. 
[14] A. Meshram, A. Kumar, and N. Srivastava, "Gas chromatography-mass spectrometry (GC-MS) analysis of alkaloids isolated from Epipremnum aureum (linden and andre) bunting," International Journal of Pharma Sciences and Research, vol. 6, no. 2, pp. 337-342, 2015.

[15] L. R. Tembrock, C. D. Broeckling, A. L. Heuberger, M. P. Simmons, F. R. Stermitz, and J. M. Uvarov, "Employing two-stage derivatisation and GC-MS to assay for cathine and related stimulant alkaloids across the celastraceae," Phytochemical Analysis, vol. 28, no. 4, pp. 257-266, 2017.

[16] K. László and V. Szke, "HPLC-ESI-MS/MS of brain neurotransmitter modulator lobeline and related piperidine alkaloids in Lobelia inflata L," Journal of Mass Spectrometry, vol. 50, no. 5, pp. 727-733, 2015.

[17] I. Rhee and K.-J. Paeng, "Simultaneous determination of betaine and choline using derivatization by HPLC with UV detection," Analytical Science and Technology, vol. 28, no. 2, pp. 112-116, 2015.

[18] U. A. Attar and S. G. Ghane, "In vitro antioxidant, antidiabetic, antiacetylcholine esterase, anticancer activities and RPHPLC analysis of phenolics from the wild bottle gourd (Lagenaria siceraria (Molina) Standl.)," South African Journal of Botany, vol. 125, pp. 360-370, 2019.

[19] U. G. Spizzirri, D. Restuccia, M. Curcio, O. I. Parisi, F. Iemma, and N. Picci, "Determination of biogenic amines in different cheese samples by LC with evaporative light scattering detector," Journal of Food Composition and Analysis, vol. 29, no. 1, pp. 43-51, 2013.

[20] Y.-X. Yang, C.-L. Mu, J.-F. Zhang, and W.-Y. Zhu, "Determination of biogenic amines in digesta by high performance liquid chromatography with precolumn dansylation," Analytical Letters, vol. 47, no. 8, pp. 1290-1298, 2014.

[21] A. Herrero, S. Sanllorente, C. Reguera, M. C. Ortiz, and L. A. Sarabia, "A new multiresponse optimization approach in combination with a D-Optimal experimental design for the determination of biogenic amines in fish by HPLC-FLD," Analytica Chimica Acta, vol. 945, pp. 31-38, 2016.

[22] F. Martin, C. Gimenez, P. Fontannaz, T. Kilinc, E. CamposGiménez, and D. Dowell, "Choline in infant formula and adult/pediatric nutritional formula by ultra high-performance liquid chromatography/tandem mass spectrometry: AOAC first action 2012.18," Journal of AOAC International, vol. 96, no. 6, pp. 1396-1399, 2013.

[23] W. Jing, J. J. Thompson, W. A. Jacobs, and L. M. Salvati, "Determination of free and total carnitine and choline in infant formulas and adult nutritional products by UPLC/MS/ MS: single-laboratory validation, first action 2014.04," Journal of AOAC International, vol. 98, no. 5, pp. 1395-1406, 2015.

[24] D. J. Ellingson, J. J. Shippar, and J. M. Gilmore, "Determination of free and total choline and carnitine in infant formula and adult/pediatric nutritional formula by liquid chromatography/tandem mass spectrometry (LC/MS/MS): singlelaboratory validation, first action 2015.10," Journal of AOAC International, vol. 99, no. 1, pp. 204-209, 2016.

[25] G. H. Gu, D. J. Yang, S. Y. Wang et al., "Simultaneous determination of eleven alkaloids in Corydalis decumbens by HPLC with diode-array detection," Acta Chromatographica, vol. 29, no. 1, pp. 121-134, 2017.

[26] Z. Xun, D. Liu, R. Huang et al., "Simultaneous determination of eight alkaloids and oleandrin in herbal cosmetics by dispersive solid-phase extraction coupled with ultra high performance liquid chromatography and tandem mass spectrometry," Journal of Separation Science, vol. 40, no. 9, pp. 1966-1973, 2017.
[27] M.-x. Su, M. Song, D.-s. Yang, J.-f. Shi, B. Di, and T.-j. Hang, "Simultaneous LC-MS/MS determination of five tripterygium pyridine alkaloids in dog plasma and its application to their pharmacokinetic study after oral administration of tripterygium glycosides tablets," Journal of Chromatography B, vol. 990, pp. 31-38, 2015.

[28] A. Lin, X. Su, D. She, K. Qiu, Q. He, and Y. Liu, "LC-MS/MS determination and comparative pharmacokinetics of strychnine, brucine and their metabolites in rat plasma after intragastric administration of each monomer and the total alkaloids from Semen Strychni," Journal of Chromatography B, vol. 1008, pp. 65-73, 2016.

[29] W. W. Zhang, X. R. Wang, S. P. Yang et al., "“Simultaneous quantification of five biogenic amines based on LC-MS/MS and its application in honeybee venom from different subspecies," Biomedical Chromatography, vol. 34, p. 4740, 2020.

[30] N. Yoshioka, S. Akamatsu, T. Mitsuhashi, C. Todo, M. Asano, and Y. Ueno, "A simple method for the simultaneous determination of mushroom toxins by liquid chromatographytime-of-flight mass spectrometry," Forensic Toxicology, vol. 32, no. 1, pp. 89-96, 2014.

[31] J.-H. Huo, X.-W. Du, G.-D. Sun, W.-T. Dong, and W.-M. Wang, "Identification and characterization of major constituents in Juglans mandshurica using ultra performance liquid chromatography coupled with time-of-flight mass spectrometry (UPLC-ESI-Q-TOF/MS)," Chinese Journal of Natural Medicines, vol. 16, no. 7, pp. 525-545, 2018.

[32] L.-W. Chen, Q. Wang, K.-M. Qin et al., "Chemical profiling of Qixue Shuangbu tincture by ultra-performance liquid chromatography with electrospray ionization quadrupole-time-offlight high-definition mass spectrometry (UPLC-QTOF/ MS)," Chinese Journal of Natural Medicines, vol. 14, no. 2, pp. 141-146, 2016. 